\title{
E-Cadherin is Critical for SC1-Induced Colony Growth of F9 Embryonic Carcinoma Cells
}

\author{
Juan Du ${ }^{a, c, d}$ Yongyan Wu ${ }^{b, c, d}$ Yingying Liu ${ }^{a, c}$ Zhiying Aia, ${ }^{a, c}$ Haibo Wu $u^{b, c}$ \\ Xiaoyan Shia,c Zekun Guo ${ }^{\mathrm{b}, c}$ Yong Zhang ${ }^{\mathrm{b}, \mathrm{c}}$
}

${ }^{a}$ College of Life Sciences, Northwest A\&F University, ${ }^{b}$ College of Veterinary Medicine, Northwest A\&F University, 'Key Laboratory of Animal Biotechnology, Ministry of Agriculture, Northwest A\&F University, Yangling, Shaanxi, China; ${ }^{\mathrm{d}}$ These authors contributed equally to this work

\section{Key Words}

$\mathrm{SC} 1$ (pluripotin) • Colony growth • E-cadherin • Pluripotency • F9 embryonic carcinoma cells

\begin{abstract}
Background: Colony morphology of embryonic stem (ES) cells contributes to the maintenance of undifferentiated ES cells. Small molecule 3,4-dihydropy-rimido[4,5-d]pyrimidine (SC1), an inhibitor of ERK1- and RasGAP-dependent signaling pathways, can maintain the compact colony morphology of ES cells. However, information on the influence of SC1 on cell morphological change remains lacking. Methods: In this study, mouse ES cells J1 and embryonic carcinoma (EC) cells F9 were cultured in SC1-containing medium to determine the effect of SC1 on cell morphology. Results: SC1 promotes a more compact morphology of J1 mouse ES cells and induces colony growth of F9 EC cells. Furthermore, the cell adhesion protein E-cadherin is a downstream target of SC1, and E-cadherin is critical for SC1-mediated colony growth of $\mathrm{F} 9$ EC cells. Conclusions: SC1 maintains and induces compact colony morphology of pluripotent cells, and its downstream target, E-cadherin, is involved in the colony phenotype of F9 EC cells. These results explored the potential role of $\mathrm{SC} 1$ in morphological change and gene expression in pluripotent cells.
\end{abstract}


Du et al.: SC1 Induced Colony Growth of F9 EC Cells

\section{Introduction}

Embryonic stem (ES) cells derived from the inner cell mass of the blastocyst can selfrenew indefinitely and differentiate into all three germ layers. These unique properties of ES cells make them potential materials for various diseases treatment [1-3]. To apply ES cells in disease treatment, stem cell regulatory mechanisms and the methods directing certain cell lineage derivation from the ES cells must be understood. Previous reports indicate that fine-tuning endogenous signaling pathways by recombinant protein or small molecules can maintain the self-renewal and pluripotency of ES cells. Activation of signal transducer and activator of transcription 3 by leukemia inhibitory factor (LIF) mediates the self-renewal of ES cells [4, 5], whereas BMP4 supports self-renewal of ES cells by inhibiting ERK and p38 mitogen-activated protein kinase [6]. However, the regulating networks involved in ES cell self-renewal and pluripotency maintenance are complex and information on them is lacking. In vitro, ES cells cannot maintain their undifferentiated state, and thus, are usually maintained and expanded on feeder cells supplied with serum and exogenous factors, such as basic fibroblast growth factor for human ES cells [7], and LIF for mouse ES cells (mESCs) [8]. When ES cells are used for tissue or cell therapies, feeder cells may contaminate the ES cells. Addition of exogenous factors to feeder cells complicates the effects of exogenous factors on ES cells because they may function indirectly by regulating of the feeder cells. To solve these problems, feeder-free and serum-free ES cell culture conditions have been studied in recent years [9-11].

Small molecules that target specific proteins or signaling pathways have been shown useful for revealing the ES cell regulatory mechanisms and cell fate determination [12]. Small molecules can support ES cell self-renewal under serum-free and feeder-free conditions [9]. Chen and colleagues demonstrated that SC1 (also referred to as pluripotin) can maintain selfrenewal and compact colony morphology under feeder-free conditions and in the absence of other exogenous factors conditions [13]. This study showed that SC1 directly binds to and inhibits RasGAP and ERK1/2 activation. RasGAP is a GTPase-activating protein that blocks ES cell self-renewal indirectly by inhibiting RasGTP-activated phosphatidylinositol 3-kinase (PI3K) $[14,15]$. Binding of SC1 to RasGAP increases RasGTP levels and further promotes PI3K-initiated ES cell self-renewal. ERK1/2 participates in the development process of ES cells, and phosphorylation of ERK1/2 is indispensable in initiating ES cell differentiation [16, 17]. Binding of SC1 to ERK1/2 directly blocks ERK1/2 phosphorylation and subsequently inhibits ES cell differentiation. Recent studies have shown that SC1 can efficiently promote the derivation of ES cell from a refractory strain when combined with LIF, and an SC1-based mESCs derivation method was generated $[18,19]$. Moreover, SC1 can replace LIF to maintain mESCs growth under mouse embryonic fibroblast feeder conditions [20]. As of this writing, although the signaling pathway responses to SC1 have been reported, information on the downstream effectors and target genes regulated by SC1 is lacking.

Transcription factors Nanog, Oct4, Sox2 and Klf4 regulate thousands of ES cell-specific gene expression by binding to their promoter directly; hence, the four factors have been considered the markers of undifferentiated ES cells [21-24]. Recently, E-cadherin, an important cell-to-cell contact protein, was found to participate in a wide range of mESCs activities, and has been used as a marker to identify and select the induced pluripotent stem cells [25-27]. In human and mouse ES cells, E-cadherin expression is downregulated when the cells were processed for early differentiation through an Epithelial-mesenchymal transition (EMT) process that is important for embryonic tissue remodeling [28, 29]. Under EMT, ES cells at the periphery of ES colonies gradually lost E-cadherin expression, which is correlated with the morphological change and motility increase in the cells, and the periphery cells of ES colonies acquire a mesenchymal-like phenotype and gradually expand until a confluence is achieved [30]. Unique interaction between E-cadherin and small GTPase Rap1 in human ES cells can enhance human ES cells self-renewal and colony formation, when E-cadherin expression is blocked, degradation of Rap1 is induced and ES clonogenic capacity is downregulated [31]. Furthermore, previous reports suggest that increased E-cadherin 
expression can enhance the compact colony phenotype and undifferentiated state of mESCs $[28,32]$, which occurs even when E-cadherin is overexpressed in feeder cells or is coated with dishes [33, 34]. During the early embryonic development of stem cells, deletion of E-cadherin can induce the embryonic failure to form an compact embryonic blastocyst [35]. The pivotal roles of E-cadherin in stem cell biology have also been demonstrated in the process of induced pluripotent stem (iPS) cell generation, the Yamanaka factors couldn't reprogram E-cadherin deletion mouse fibroblasts to induced pluripotent stem cells [36], and E-cadherin overexpression significantly enhanced the efficiency of iPS cell generation [37]. Thus, E-cadherin and its tight adhesion function are indispensable for the undifferentiated state of ES cells. The functional role of E-cadherin in ES cells has been explored, but information on the regulatory mechanisms of E-cadherin in stem cells is still lacking.

In this study, we used SC1 to support the undifferentiated state of J1 mESCs and mouse F9 embryonic carcinoma (EC) cells in vitro. We found that SC1 can maintain the compact growth of J1 mESCs and initiate a specific colony formation of F9 EC cells. Our data demonstrated that E-cadherin is a downstream target of SC1, and E-cadherin is involved in colony formation of F9 EC cells. These findings implied that SC1 promotes colony growth in F9 EC cells, at least partly by modulating E-cadherin.

\section{Materials and Methods}

\section{Reagents}

Unless otherwise indicated, reagents were purchased from Sigma Chemical Co. (St. Louis, MO, USA). Primary antibodies: mouse anti-Oct4, rabbit anti-Nanog, goat anti-E-cadherin and rabbit anti- $\beta$-catenin were purchased from Santa Cruz Biotechnology Inc. (Santa Cruz, CA, USA). DyLight 549-, 488- and Cy3-labeled secondary antibodies, secondary antibodies for Western blot, DAPI, buffers used for immunofluorescence, BCIP/NBT Alkaline Phosphatase (AP) Color Development Kit, and WST-1 Cell Proliferation and Cytotoxicity Assay Kit were purchased from Beyotime Institute of Biotechnology (Jiangsu, China). SC1 was purchased from Santa Cruz Biotechnology Inc. (Santa Cruz, CA, USA).

\section{Cell culture and SC1 treatment}

J1 ES cells were purchased from ATCC (Manassas, USA), maintained on 0.1\% gelatin-coated dishes, and grown in OriCell ${ }^{\mathrm{TM}}$ Strain C57BL/6 Mouse ES Cell Growth Medium (Cyagen Biosciences, Guangzhou, China). Mouse F9 EC cells were purchased from the bank of the Chinese Academy of Sciences (Shanghai, China) and maintained as described previously [38]. The F9 EC cells were maintained on $0.1 \%$ gelatincoated plates in Dulbecco's modified Eagle's medium supplemented with $10 \%$ fetal bovine serum. Cells were maintained at $37^{\circ} \mathrm{C}$ and $5 \% \mathrm{CO}_{2}$.

SC1 dissolved in dimethyl sulfoxide (DMSO) was stored at $-20^{\circ} \mathrm{C}$ with a concentration of $10 \mathrm{mM}$. When treating cells, SC1 was added directly to the pre-warmed cell culture medium.

Cell viability and colony formation assay

Cell viability was evaluated by WST-1 assay according to the manufacturer's instructions (Beyotime Institute of Biotechnology, Jiangsu, China). Replicate wells of 3200 cells in a $100 \mu \mathrm{l}$ medium in the absence or presence of SC1 were plated into 96 -well plates and incubated at $37{ }^{\circ} \mathrm{C}$ in $5 \% \mathrm{CO}_{2}$.

To analyze the effects of SC1 on F9 EC cell colony formation. F9 EC cells were plated into 12-well plates $(8,000$ cells per well). Cells were cultured for $24 \mathrm{~h}$ in the absence or presence of the SC1. After 24 $\mathrm{h}$, cells were stained with AP substrate, and the colonies were counted. DMSO-treated cells were grown in monolayer, and we counted the cells that connected with each other and showed no clear interspaces as one colony.

\section{Immunofluorescence Staining and AP Staining}

Cells were fixed and blocked according to the manufacturer's instructions (Beyotime institute of biotechnology, Jiangsu, China). For immunofluorescence staining, cells were incubated with the indicated primary antibodies overnight at $4{ }^{\circ} \mathrm{C}$. After washing with wash buffer three times, cells were incubated with 
DyLight 549, DyLight 488 or Cy3-labeled secondary antibodies for $1 \mathrm{~h}$ at $37^{\circ} \mathrm{C}$. Nuclei were stained with DAPI.

For AP staining, fixed cells were incubated in a BCIP/NBT working solution for $10 \mathrm{~min}$ in the dark, and then washed four times with distilled water.

RT-PCR and quantitative real time PCR ( $q P C R)$

Total RNAs were extracted using TRIzol ${ }^{\circledR}$ reagent (Invitrogen, CA, USA) according to the manufacturer's instructions. Isolated RNAs were reverse transcribed into cDNA using SYBR PrimeScript ${ }^{\mathrm{TM}}$ RT-PCR Kit (Takara, Dalian, China). For qPCR, a reaction mixture was assembled according to the protocol of SYBR ${ }^{\circledast}$ Premix Ex Taq ${ }^{\text {TM }}$ (Takara). The conditions for qPCR were: $30 \mathrm{~s}$ at $95{ }^{\circ} \mathrm{C}, 40$ cycles for $5 \mathrm{~s}$ at $95{ }^{\circ} \mathrm{C}$ and $30 \mathrm{~s}$ at $60^{\circ} \mathrm{C}$. The comparative $\mathrm{Ct}$ method was used to calculate the relative quantity of the target gene mRNA, which was normalized to glyceraldehyde 3-phosphate dehydrogenase $(G A P D H)$ and relative to the calibrator. The qPCR primer sequences used for Klf4, Nanog, Oct4, and Sox2 were described previously [39]. The qPCR primer sequences for E-cadherin and $\beta$-catenin were as follows: E-cadherin (sense) 5'-CAGGTCTCCTCATGGCTTTGC-3' and (anti-sense) 5'-CTTCCGAAAAGAAGGCTGTCC-3'; $\beta$-catenin (sense) 5'-ACCTACTCCCTCCTTTCTACTCC-3' and (anti-sense) 5'-CCGCAGGGCAGGATAAAAAGT-3'.

\section{Knockdown of E-cadherin}

Cells were transfected with small interference RNAs (siRNA) target mouse E-cadherin using Lipofectamine ${ }^{\mathrm{TM}}$ RNAi MAX (Invitrogen) according to the manufacturer's instructions. The siRNAs were synthesized by GenePharma (Shanghai, China), using the following sequences ( 5 ' to 3'): 1634, sense, GGGUCAGGAAAUCACAUCUTT, antisense, AGAUGUGAUUUCCUGACCCTT; 2042, sense, CUGGACCAUUGAGUAUAAUTT, antisense, AUUAUACUCAAUGGUCCAGTT; 2203, sense, CGGUCAACAACUGCAUGAATT, antisense, UUCAUGCAGUUGUUGACCGTT; Negative control (NC) siRNA, which has a non-targeting sequence, was provided by GenePharma (Shanghai, China).

\section{Western blot analysis}

For Western blot, $8 \%$ and $12 \%$ acrylamide gels were used. Separated proteins were transferred onto polyvinylidene difluoride membranes (Millipore, Bedford, MA, USA) for $3 \mathrm{~h}$ at $250 \mathrm{~mA}$. The membranes were then blocked in 10\% non-fat milk prepared with tris-buffer saline and Tween 20 (TBST) at pH 7.0 for $2.5 \mathrm{~h}$. Subsequently, the blocked membranes were incubated with primary antibody (dilution 1:1000) at 4 ${ }^{\circ} \mathrm{C}$ overnight. After washing three times with TBST, the membranes were further incubated with secondary antibody (dilution 1:1000) for approximately $2 \mathrm{~h}$ at room temperature. After washing three times for 15 min each time, the immunoblots were revealed by autoradiograph using SuperSignal West Pico substrate (Pierce/Thermo Scientific, Rockford, IL, USA).

\section{Plasmid construction}

The pCDH-CMV-E-cadherin-EF1-copGFP plasmid was generated by amplifying the fulllength mouse open reading frame E-cadherin sequence from the cDNA of F9 EC cells and subsequently cloned into the pCDH-CMV-MCS-EF1-copGFP vector. Primer sequences for E-cadherin amplification are (sense) 5'-TGTCTAGAGCCACCATGGGAGCCCGGTGCCGCAGCTT-3' and (anti-sense) 5'-AGAGCGGCCGCTTAGTCGTCCTCGCCACCGCCGTACATGTCCGCCA-3' (the underline sequences are restriction sites).

shRNA plasmid target Nanog was constructed by inserting indicated sequence to pSilencer2.1-U6 hygro vector (Applied Biosystems, Foster City, CA, USA) according to the manufacturer's instructions. Sequences of the shRNA template for Nanog (sh-Nanog) were: forward: GATCCGCACCACACCTGGCATATATTTCAAGAGAATATATGCCAGGTGTGGTGTTTTTTGGAAA; reverse: AGCTTTTCCAAAAAACACCACACCTGGCATATATTCTCTTGAAATATATGCCAGGTGTGGTGCG; Sequences of the negative control shRNA plasmid (sh-scramble) were: forward: GATCCGAAAGTAGAGCGCAGAACTTTCAAGAGAAGTTCTGCGCTCTACTTTCTTTTTTGGAAA; reverse: AGCTTTTCCAAAAAAGAAAGTAGAGCGCAGAACTTCTCTTGAAAGTTCTGCGCTCTACTTTCG.

For E-cadherin overexpression and Nanog knockdown, F9 EC cells were transfected with indicated plasmids using Lipofectamine ${ }^{\mathrm{TM}} 2000$ Transfection Reagent (Invitrogen). 


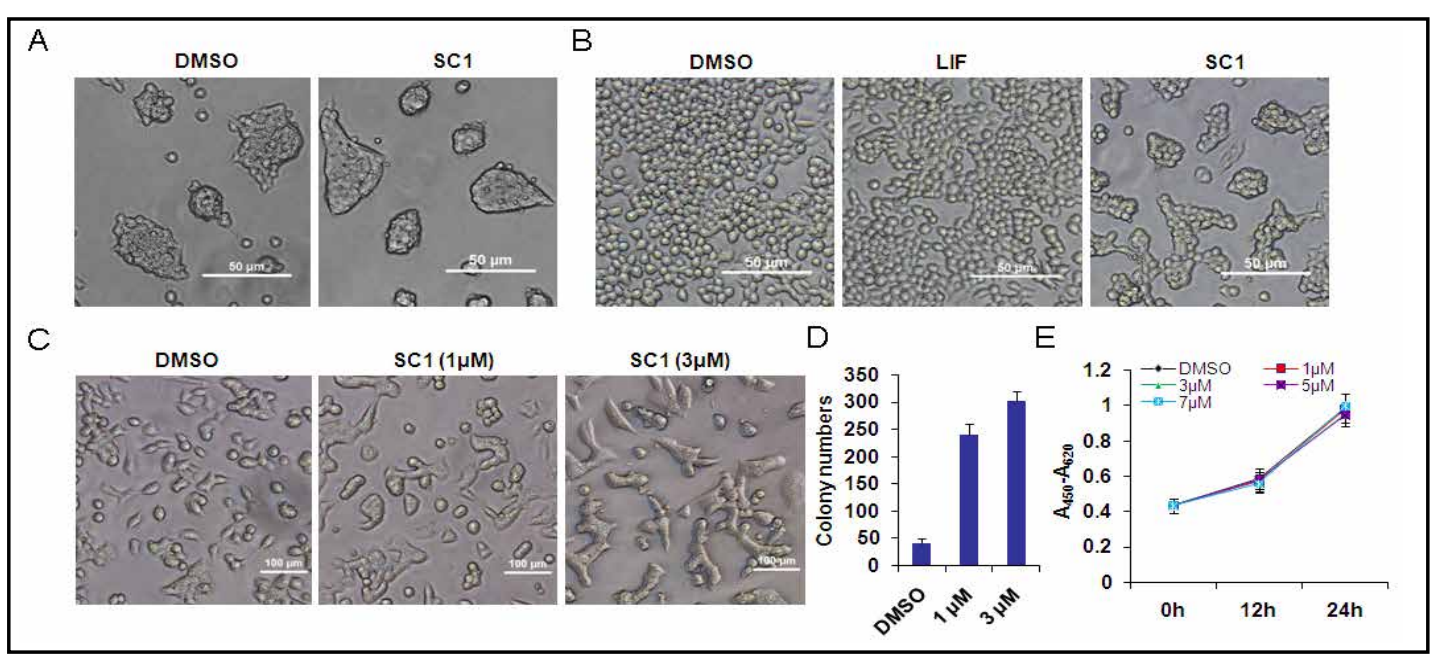

Fig. 1. SC1-induced morphological change of F9 EC cells. A: Morphology of J1 mESCs cultured in medium with DMSO (used as control) or $3 \mu \mathrm{M} \mathrm{SC1}$ for $24 \mathrm{~h}$. B: Morphology of F9 EC cells treated with DMSO, LIF $(1000 \mathrm{U} / \mathrm{mL})$ or SC1 $(3 \mu \mathrm{M})$ for $24 \mathrm{~h}$. C: Morphology of F9 cells that treated with DMSO, $1 \mu \mathrm{M}$ SC1 and $3 \mu \mathrm{M}$ SC1 for 3 h. D: Colony numbers of F9 EC cells which treated with DMSO, $1 \mu$ M SC1, or $3 \mu$ M SC1 for 24 h. E: Cell viability assay of F9 EC cells treated with various concentration of SC1. F9 EC cells were treated with indicated concentrations of SC1, and the cell viability was determined by WST-1 kit at the time points of 0 , 12 and $24 \mathrm{~h}$ respectively. $\mathrm{A}_{450}, \mathrm{~A}_{620}$ : absorbance at $450 \mathrm{~nm}$ and $620 \mathrm{~nm}$.

E-cadherin promoter (-548 to +172 relative the TSS) [40] was amplified from the genome DNA of F9 cells. Primer sequences for E-cadherin promoter amplification were (sense) 5'-CTGGGTACCAAAAAGTCACCAAACAAAATAAAACCGTCGGAG-3' and (anti-sense) 5'-ATCCTCGAGCAGCAGGAGCAGGAGCGCGGAAAAGCTG-3'. The E-cadherin promoter was inserted into pGL4.10 reporter vector (Promega, Madison, WI), and designated as pGL4.10-P E-cadherin.

Luciferase reporter assays

Luciferase assays were performed using a Dual-Luciferase Reporter Assay System (Promega, Madison, WI, USA) according to the manufacturer's instructions. Briefly, cells were co-transfected with luciferase reporter plasmids (experimental reporter pGL4.10- P E-cadherin or control reporter pGL4.10) and the Renilla luciferase plasmid pRL-SV40 (Promega) using Lipofectamine ${ }^{\mathrm{TM}} 2000$ Reagent (Invitrogen). After 12 $\mathrm{h}$, transfected cells were treated with $3 \mu \mathrm{M}$ SC1 or an equal volume of DMSO for an additional $24 \mathrm{~h}$. Then, the cells were lysed in $1 \times$ passive lysis buffer for $15 \mathrm{~min}$ with shaking. Luciferase activity in cell lysates was examined on a VICTOR X5 multilabel plate reader (PerkinElmer, Cetus, Norwalk, USA) and normalized to Renilla luciferase.

\section{Statistical Analysis}

All experiments described were repeated three times. Data were presented as the mean \pm standard deviation. For statistical analysis, Student's t-test was used, and statistical significance was denoted as $\mathrm{P}<$ 0.05 .

\section{Results}

SC1 induces morphological change of F9 EC cells

As shown in Fig. 1A, J1 mESCs cultured in SC1-containing medium acquired a smoother boundary and a more compact morphology compared with the control cells which were treated with the solvent DMSO. Based on the above observation, we hypothesized that SC1 can promote aggregate growth of cells. F9 mouse EC cells and NIH3T3 mouse embryo fibroblasts, which are mouse cell lines that have different origins but both grow in monolayer, were treated 
Fig. 2. SC1 treatment maintains pluripotency of F9 EC cells. A: AP staining of F9 EC cells treated with DMSO or $3 \mu \mathrm{M} \mathrm{SC1}$ for $24 \mathrm{~h}$. B: qPCR analysis of the key pluripotency factors Oct4, Sox2 and Klf4. F9 EC cells were treated with DMSO, $1 \mu \mathrm{M}$ SC1 or $3 \mu \mathrm{M}$ SC1 for 6 and 24 h. C: Western blot analysis of Oct4 and Sox 2 protein levels after $24 \mathrm{~h}$ treatment with DMSO or SC1 at the indicated concentration. D: qPCR analysis of Nanog transcripts. Cells were treated with DMSO, $1 \mu \mathrm{M}$ SC1 or $3 \mu \mathrm{M}$ SC1 for 6 and $24 \mathrm{~h}$. E: Western blot analysis of Nanog protein. Cells were treated DMSO, $1 \mu \mathrm{M}$ and 3 $\mu \mathrm{M}$ SC1 for $24 \mathrm{~h}$. DMSO-treated cells were used as control. F: Immunofluorescence staining of Nanog (green), Oct4 (red), and DNA (DAPI, blue). F9 EC cells were treated with DMSO or $3 \mu \mathrm{M}$ SC1 for $24 \mathrm{~h}$.

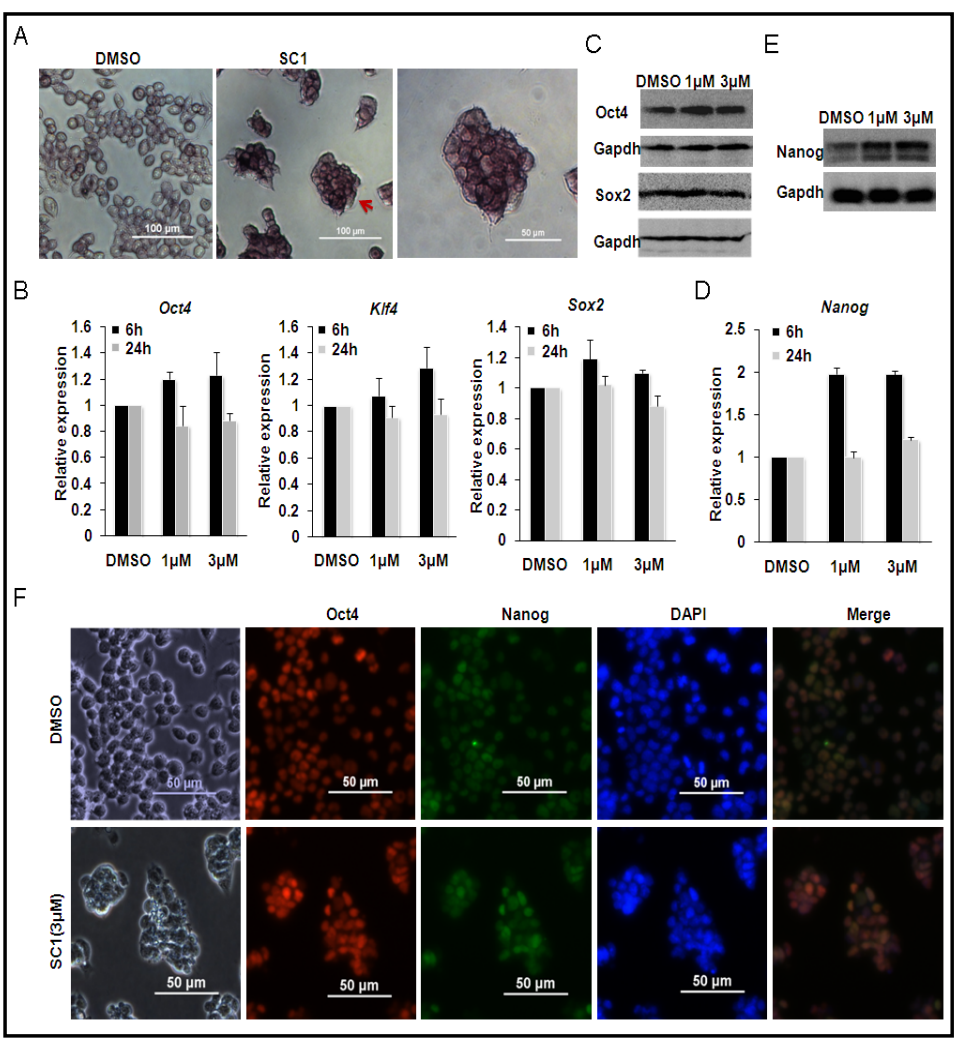

with SC1 and DMSO. After 24 h, F9 EC cells incubated in DMSO or LIF-containing medium still developed in monolayer similar to untreated cells. In contrast, F9 EC cells cultured in SC1-containing medium accumulated and formed three-dimensional clusters (Fig. 1B). This result demonstrates that SC1 other than LIF causes F9 cell aggregation. Considering that the morphology similarity of the F9 EC cell clusters with mESCs, we designated the SC1-induced F9 clusters as colonies thereafter. At $3 \mathrm{~h}$ after treatment, the accumulation of F9 EC cells in $3 \mu \mathrm{M}$ SC1-containing medium was higher than that in $1 \mu \mathrm{M}$ SC1-containing medium (Fig. 1C). Twenty-four hours after treatment, $1 \mu \mathrm{M}$ and $3 \mu \mathrm{M}$ SC1 both induced clearly colonies of F9 EC cells, but the colony number in $3 \mu \mathrm{M}$ SC1-treated cells was higher than that of $1 \mu \mathrm{M}$ SC1-treated cells (Fig. 1D). Notably, F9 EC cell morphological change caused by SC1 did not alter its proliferation or cell viability (Fig. 1E). For NIH3T3 cells, no obvious morphological changes were observed after SC1 treatment $(0.1 \mu \mathrm{M}$ to $1 \mu \mathrm{M})$, and higher concentration of SC1 $(2 \mu \mathrm{M}$ to $5 \mu \mathrm{M})$ was toxic to NIH3T3 cells, as cell mortality was observed during $24 \mathrm{~h}$ treatment (data not shown).

The above results indicated that SC1 can promote aggregation and colony formation of cells, but this capability of SC1 is cell type-dependent and not applicable to NIH3T3 cells.

SC1-induced morphological change of F9 EC cells did not downregulate the expression of pluripotency factors

SC1 was used for pluripotency maintenance. Therefore, whether or not F9 colonies caused by SC1 addition still maintained pluripotency was determined. Undifferentiated pluripotent cells showed elevated levels of AP activity, and therefore AP staining was used to test pluripotency. The results showed that AP activity of SC1-treated F9 EC cells was higher than that of DMSO-treated cells (Fig. 2A). SC1 did not affect the proliferation of F9 EC cells, and thus, SC1-induced increase in AP staining in F9 EC cells was not caused by cell number increase but by the colony formation of the cells. Quantitative real-time PCR (qPCR) examination of three key pluripotency markers showed that Klf4, Oct4 and Sox2 expression did not changed significantly after $24 \mathrm{~h} \mathrm{SC1}$ treatment, but a slight up-regulation after $6 \mathrm{~h}$ 


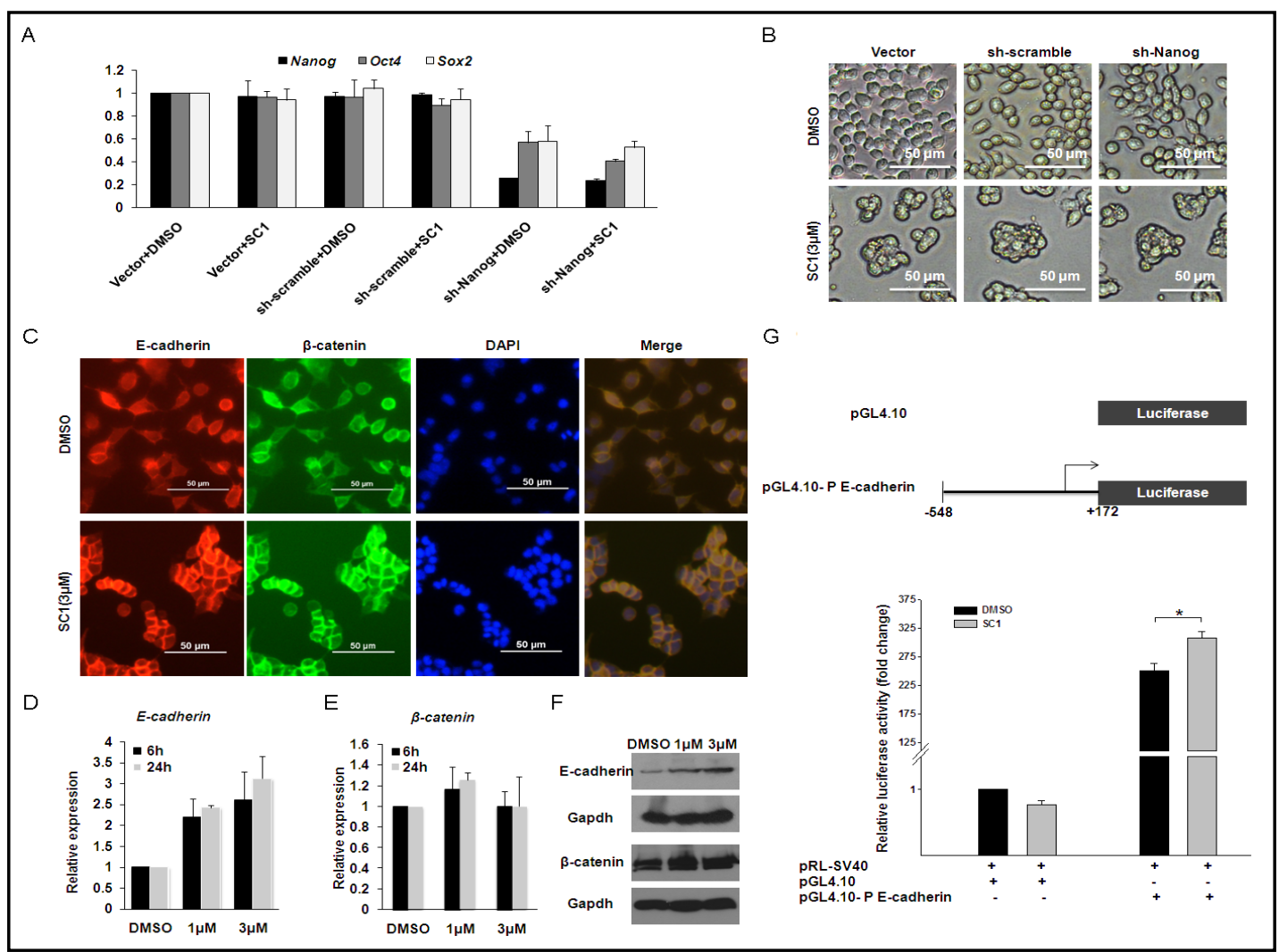

Fig. 3. Effects of SC1 on E-cadherin and $\beta$-catenin expression and subcellular localization. A: qPCR analysis of Nanog, Oct4 and Sox2 transcripts. 12 hours after pSilencer2.1-U6 hygro (Vector), sh-scramble or sh-Nanog transfection, F9 EC cells were treated with DMSO or $3 \mu \mathrm{M} \mathrm{SC1}$ for another $24 \mathrm{~h}$. B: Morphology of F9 EC cells. Cells pre-transfected with indicated plasmids were treated with DMSO and $3 \mu \mathrm{M} \mathrm{SC1} \mathrm{for} 24 \mathrm{~h}$. C: Immunofluorescence staining of E-cadherin (red), $\beta$-catenin (green), and DNA (DAPI, blue) in F9 EC cells that treated with DMSO or $3 \mu \mathrm{M} \mathrm{SC1}$ for $24 \mathrm{~h}$. D and E: qPCR analysis of E-cadherin (D) and $\beta$-catenin (E) gene transcripts in DMSO- or SC1-treated F9 EC cells. F9 EC cells were treated with various concentrations of SC1 for 6 hours or $24 \mathrm{~h}$. F: Western blot analysis of E-cadherin and $\beta$-catenin after $24 \mathrm{~h}$ of treatment with DMSO or SC1. G: Upper panel: a schematic for E-cadherin promoter reporter. Bottom panel: dual-luciferase reporter assay of E-cadherin promoter activity. $12 \mathrm{~h}$ after transfection, $3 \mu \mathrm{M}$ SC1 or an equal volume of DMSO was added to the F9 EC cells culture medium for an additional $24 \mathrm{~h}$ and then the luciferase activity of E-cadherin promoter was determined. *: $\mathrm{p}<0.05$.

treatment was observed (Fig. 2B). Western blot analysis of the protein levels after $24 \mathrm{~h}$ of treatment showed consistent results (Fig. 2C). Transcription level of Nanog was significantly upregulated after $6 \mathrm{~h}$ of SC1 treatment, but reverted to basal level after $24 \mathrm{~h}$ of treatment (Fig. 2D). However, Nanog protein maintained higher level of expression after $24 \mathrm{~h} \mathrm{SC1-treatment}$ compared with control cells (Fig. 2E). Furthermore, immunofluorescence indicated that SC1 did not alter the subcellular localization of Nanog and Oct4: both of them distributed in the nucleus as control cells (Fig. 2F).

Collectively, the results suggest that colony formation of F9 EC cells induced by SC1 maintained pluripotency because elevated AP activity and pluripotency marker Nanog protein were detected in SC1-treated cells.

\section{SC1 promotes E-cadherin expression in F9 EC cells}

Since the expression of Nanog was increased transiently after SC1 treatment, it is possible that Nanog is critical for SC1 induced F9 EC cells colony formation. To address this question, Nanog was knocked down by shRNA plasmid, the RNAi efficiency was confirmed 


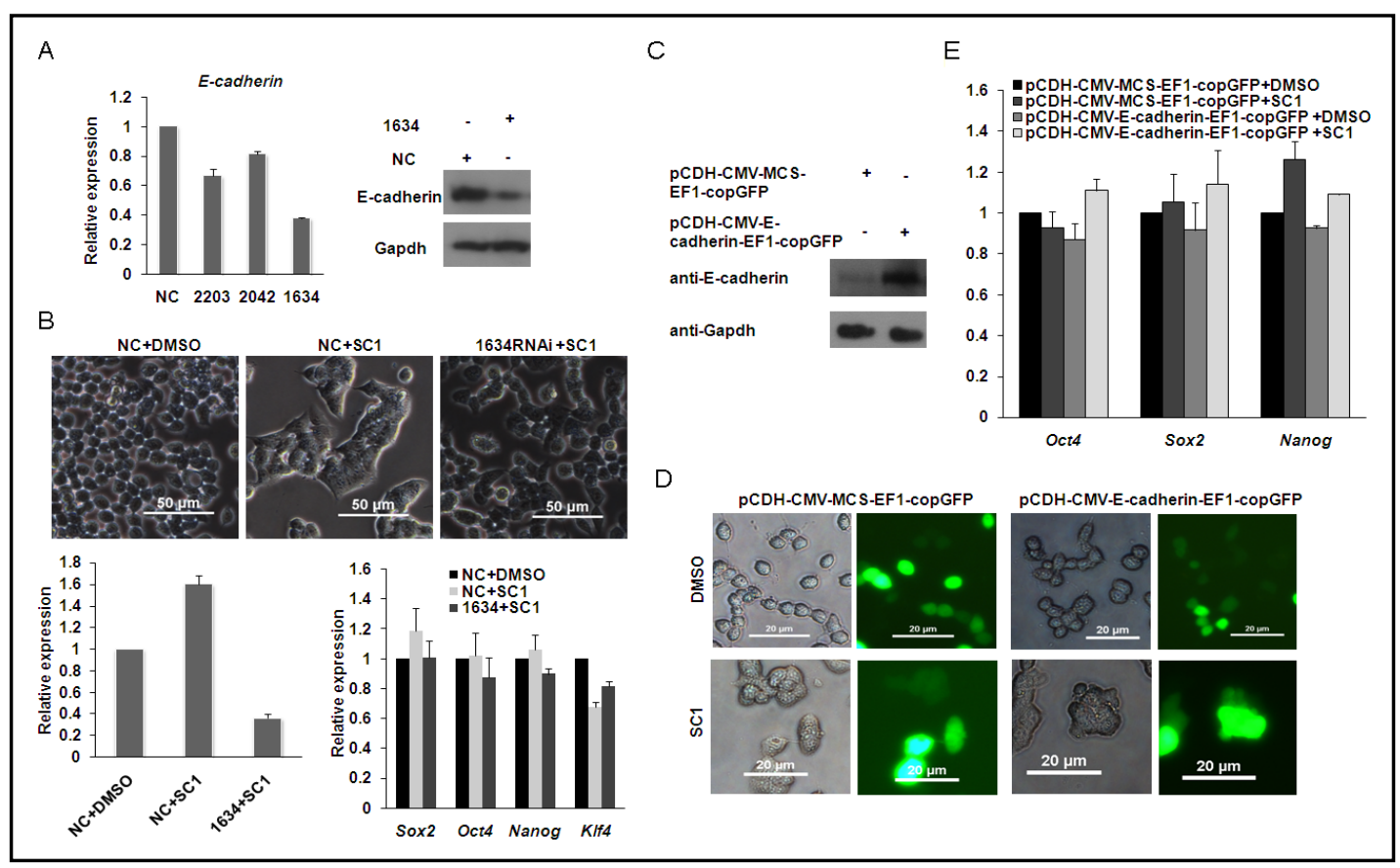

Fig. 4. E-cadherin is critical but insufficient for colony formation of F9 EC cells. A: qPCR and Western blot analysis of E-cadherin expression in F9 EC cells transfected with siRNAs target E-cadherin or negative control siRNA. B: Morphology and pluripotency factors changes of E-cadherin knockdown F9 EC cells. After 12 $\mathrm{h}$ transfection, F9 EC cells with the 1634 siRNA or negative control siRNA were culture in DMSO or $3 \mu \mathrm{M}$ SC1 contain medium for an additional $24 \mathrm{~h}$. Transcript levels of E-cadherin, Sox2, Oct4, Nanog and Klf4 were determined by qPCR experiment. C: Western blot analysis of E-cadherin expression in $36 \mathrm{~h}$ pCDH-CMV-MCSEF1-copGFP- and pCDH-CMV-E-cadherin-EF1-copGFP-transfected F9 EC cells. D: Morphology of F9 EC cells transfected with pCDH-CMV-MCS-EF1-copGFP and pCDH-CMV-E-cadherin-EF1-copGFP. After $12 \mathrm{~h}$ transfection, F9 EC cells were treated with DMSO or $3 \mu \mathrm{M} \mathrm{SC1}$ for $24 \mathrm{~h}$. GFP was used to examine the transfection efficiency of the vectors. E: Relative expression levels of pluripotency genes Oct4, Sox 2 and Nanog. $12 \mathrm{~h}$ after transfection, F9 EC cells with indicated plasmids were treated with DMSO and $3 \mu \mathrm{M} \mathrm{SC1}$ for additional $24 \mathrm{~h}$.

by qPCR and further confirmed by the downregulation of Oct4 and Sox2 (Fig. 3A). Notably, we found that knockdown of Nanog did not impair the effect of SC1 on the colony formation of F9 EC cells (Fig. 3B). Thus, Nanog is stimulated by SC1, it is but not the key factor involved in F9 EC cell colony formation.

SC1 treatment caused compact morphology of J1 mESCs and aggregation and colony formation of F9 EC cells, it is possible that SC1 directly enhanced cell-to-cell adhesion [41]. Cell adhesion proteins E-cadherin and $\beta$-catenin are critical for cell-to-cell contact and induced pluripotent stem cell identification [37, 42]. Hence, the subcellular localization of E-cadherin and $\beta$-catenin in F9 EC cells were examined by immunofluorescence. We found that E-cadherin and $\beta$-catenin were distributed uniformly in cell membranes of DMSOtreated cells. On the contrary, in SC1-treated cells, E-cadherin and $\beta$-catenin translocated and accumulated in the membrane where cells were in contact with each other (Fig. 3C). qPCR analysis revealed that $6 \mathrm{~h}$ after SC1 treatment, the transcription of E-cadherin was increased two-fold compared with control cells, and upregulation increased three-fold after $24 \mathrm{~h}$ of treatment (Fig. 3D). By contrast, no significant change of $\beta$-catenin transcripts was detected between cells treated with SC1 and DMSO (Fig. 3E). Western blot and immunofluorescence results confirmed that SC1 promotes E-cadherin expression in F9 EC cells (Fig.3C and 3F). The protein level of $\beta$-catenin in SC1-treated cells was higher than that of control cells after $24 \mathrm{~h}$ of treatment (Fig. 3C and 3F), which may be due to the protection provided by increased E-cadherin against GSK-3-mediated degradation by protein-to-protein interaction. 
To further confirm that SC1 promotes E-cadherin expression, a dual-luciferase reporter assay was introduced to study the effect of SC1 on transcriptional activity of E-cadherin promoter. The proximal sequence of mouse E-cadherin promoter $(-548$ to +172 relative to the transcription start site) was amplified and cloned into pGL4.10 vector (Fig. 3G, upper panel), and subsequently, the luciferase reporter experiment was performed under the condition with or without SC1. The result showed that SC1 enhanced the transcriptional activity of the E-cadherin promoter, and the relative luciferase activity in SC1-treated cells was significantly higher than in control cells (Fig. 3G, bottom panel). These results suggest that the cell adhesion protein, E-cadherin, is a direct downstream target of SC1.

\section{E-cadherin is critical but insufficient to initiate F9 EC cell colony formation}

Based on the above results, testing the role of E-cadherin and $\beta$-catenin in SC1-induced F9 EC cell colony formation is required. $\beta$-catenin knockdown and overexpression showed no significant effect on SC1-induced F9 EC colonies (data not shown). Thus, we focused on E-cadherin. Transfection of F9 EC cells with siRNA target mouse E-cadherin gene or negative control siRNA was performed, and subsequently, the knockdown efficiency was determined by qPCR and Western blot analysis. The results demonstrate that the siRNA 1634 is most efficient at reducing E-cadherin expression (Fig. 4A). We found that the knockdown of E-cadherin compromised SC1-mediated colony formation, and knockdown of E-cadherin before SC1 treatment resulted in the development of the F9 cells in a monolayer and no obvious colonies were formed (Fig. 4B). We examined the expression of pluripotency factors in E-cadherin knockdown cells compared with negative control siRNA transfected cells. E-cadherin knockdown did not significantly change the expression of pluripotency factors Sox2, Oct4, Nanog, and Klf4 (Fig. 4B). To further assess the role of E-cadherin in SC1-induced colony growth of F9 EC cells, an E-cadherin expression vector, pCDH-CMV-E-cadherin-EF1copGFP, which expresses GFP independently using the EF1 promoter, was constructed. Overexpression of E-cadherin (Fig. 4C) increased the cell-to-cell contact of F9 EC cells (Fig. 4D), however, there was no obvious colony formation in E-cadherin transfected F9 EC cells (Fig. 4D). Notably, compared to the control vector pCDH-CMV-MCS-EF1-copGFP transfected cells, E-cadherin overexpressed cells showed no significant morphological change after SC1 treatment (Fig. 4D). qPCR examination of pluripotency genes Sox2, Oct4 and Nanog showed that overexpression of E-cadherin have no influence on pluripotency genes expression (Fig. 4E).

Taken together, these results indicated that E-cadherin is involved in SC1-induced cell morphological change, but overexpression of E-cadherin alone is insufficient to initiate the colony formation of F9 EC cells. Other SC1-regulated genes or signalling is required for the colony formation of F9 EC cells.

\section{Discussion}

ES cells differentiate spontaneously when cultured in vitro. The small molecule SC1 maintains the pluripotency and colony phenotype of mESCs. Under feeder-free conditions, OG2-mES cells maintained in SC1-containing medium showed more compact colonies than cells cultured in LIF+BMP4-containing medium [13]. In this study, we found that SC1, but not LIF, promotes colony morphology in F9 EC cells (Fig. 1B). These results indicated that in addition to maintaining the undifferentiated state and compact colony morphology of mESCs, SC1 may also initiate colony growth of F9 EC cells, and this capability of SC1 is independent of LIF.

J1 mESCs were derived from the 129S4/SvJae embryos [43], F9 EC cells were isolated from testicular teratoma of $129 / \mathrm{Sv}$ mice [44]. NIH3T3 cells were derived from mouse embryonic fibroblasts [45]. The previous two cell lines are pluripotent cells. NIH3T3 cells, however, are highly differentiated cells. Our data showed that SC1 is able to maintain the compact colony phenotype of J1 mESCs and can induce the colony formation of F9 EC cells, 
whereas no similar effects were observed in NIH3T3 cells. The adhesion enhancement and colony promoting properties of SC1 are not applicable to all mouse cell types. Pre-activated pluripotent network or some genes specifically expressed in F9 and J1 mESCs are needed for the SC1-mediated colony phenotype.

Nanog is a pluripotency marker that is highly expressed in ES cells, EC cells, and embryonic germ (EG) cells, but decreased rapidly during the cell differentiation process $[46,47]$. Nanog overexpression caused F9 EC cells to adopt a compact colony shape that resembles ES cells cultured on feeder layers [48]. Thus, SC1-mediated colony formation of F9 EC cells may have been through the upregulating of Nanog expression. However, our results showed that SC1 triggered transient transcripts upregulation and protein increase of Nanog, but the Nanog amount did not affect SC1-initiated colony formation of F9 EC cells, and knockdown of Nanog did not block SC1-induced F9 EC cell colony formation (Fig. 3A and Fig. 3B). Thus, Nanog is likely a downstream gene of SC1 that contributes little to F9 EC cell morphological change.

Previous reports have shown that neutralization of E-cadherin inhibited colony formation of mouse (EG) cells [49], and that the rescue of E-cadherin in E-cadherin-/mESCs restored cell-to-cell contacts [26]. In this study, SC1 is found to promote E-cadherin expression by enhancing its transcriptional activity. Furthermore, knockdown of E-cadherin impaired SC1-induced colony formation of F9 EC cells. However, overexpression of E-cadherin alone cannot initiate the aggregate of F9 EC cells. Hence, we speculated that upregulation of E-cadherin may be a possible mechanism of SC1-induced colony formation of F9 EC cells. However, E-cadherin alone is insufficient to initiate the colony formation of the cells, and other collaborators of E-cadherin that respond to SC1 are needed to initiate and maintain the colony phenotype of F9 EC cells.

In conclusion, we explored the capability and mechanisms of SC1 to initiate the aggregate and colony formation of pluripotent F9 EC cells. The results suggested SC1-induced E-cadherin is involved in SC1-induced morphological change in F9 EC cells, and other SC1regulated genes or signalling pathways are needed to initiate the colony formation. In the future, genome-wide profiling of SC1-treated pluripotent cells is needed to identify other pivotal factors that participate in SC1-mediated colony and pluripotency maintenance.

\section{Conflicts of Interests}

The authors have declared that no conflicts of interest exist.

\section{Acknowledgements}

This work was supported by the National Natural Science Foundation of China [grant number 31172279].

\section{References}

1 Martin GR: Isolation of a pluripotent cell line from early mouse embryos cultured in medium conditioned by teratocarcinoma stem cells. Proc Natl Acad Sci USA 1981;78:7634-7638.

2 Bernstein HS, Srivastava D: Stem cell therapy for cardiac disease. Pediatric Res 2012;71:491-499.

3 Khanna A, Shin S, Rao MS: Stem Cells for the Treatment of Neurological Disorders. Cns Neurol Disord-Dr 2008;7:98-109.

- 4 Niwa H, Burdon T, Chambers I, Smith A: Self-renewal of pluripotent embryonic stem cells is mediated via activation of STAT3. Genes Dev 1998;12:2048-2060.

-5 Cartwright P: LIF/STAT3 controls ES cell self-renewal and pluripotency by a Myc-dependent mechanism. Development 2005;132:885-896. 


\section{Cellular Physiology Cell Physiol Biochem 2014;33:501-512 and Biochemistry \\ Du et al.: SC1 Induced Colony Growth of F9 EC Cells}

6 Qi X, Li TG, Hao J, Hu J, Wang J, Simmons H, Miura S, Mishina Y, Zhao GQ: BMP4 supports self-renewal of embryonic stem cells by inhibiting mitogen-activated protein kinase pathways. Proc Natl Acad Sci USA 2004;101:6027-6032.

7 Amit M, Carpenter MK, Inokuma MS, Chiu CP, Harris CP, Waknitz MA, Itskovitz-Eldor J, Thomson JA: Clonally derived human embryonic stem cell lines maintain pluripotency and proliferative potential for prolonged periods of culture. Dev Biol 2000;227:271-278.

$>8$ Smith AG, Heath JK, Donaldson DD, Wong GG, Moreau J, Stahl M, Rogers D: Inhibition of pluripotential embryonic stem cell differentiation by purified polypeptides. Nature 1988;336:688-690.

-9 Tsuji Y, Yoshimura N, Aoki H, Sharov AA, Ko MSH, Motohashi T, Kunisada T: Maintenance of undifferentiated mouse embryonic stem cells in suspension by the serum- and feeder-free defined culture condition. Dev Dyn 2008;237:2129-2138.

10 Li Z, Leung M, Hopper R, Ellenbogen R, Zhang M: Feeder-free self-renewal of human embryonic stem cells in 3D porous natural polymer scaffolds. Biomaterials 2010;31:404-412.

11 Chen L, Khillan JS: Promotion of feeder-independent self-renewal of embryonic stem cells by retinol (vitamin A). Stem Cells 2008;26:1858-1864.

12 Li W, Ding S: Small molecules that modulate embryonic stem cell fate and somatic cell reprogramming. Trends Pharmacol Sci 2010;31:36-45.

13 Chen S, Do JT, Zhang Q, Yao S, Yan F, Peters EC, Scholer HR, Schultz PG, Ding S: Self-renewal of embryonic stem cells by a small molecule. Proc Nat Acad Sci USA 2006;103:17266-17271.

14 Letso RR, Stockwell BR: Chemical biology: renewing embryonic stem cells. Nature 2006;444:692-693.

15 Rudack T, Xia F, Schlitter J, Kotting C, Gerwert K: Ras and GTPase-activating protein (GAP) drive GTP into a precatalytic state as revealed by combining FTIR and biomolecular simulations. Proc Natl Acad Sci USA 2012;109:15295-15300.

16 Nichols J, Silva J, Roode M, Smith A: Suppression of Erk signalling promotes ground state pluripotency in the mouse embryo. Development 2009;136:3215-3222.

17 Lanner F, Rossant J: The role of FGF/Erk signaling in pluripotent cells. Development 2010;137:3351-3360.

18 Yang W, Wei W, Shi C, Zhu J, Ying W, Shen Y, Ye X, Fang L, Duo S, Che J, Shen H, Ding S, Deng H: Pluripotin Combined with Leukemia Inhibitory Factor Greatly Promotes the Derivation of Embryonic Stem Cell Lines from Refractory Strains. Stem Cells 2009;27:383-389.

19 Pieters T, Haenebalcke L, Hochepied T, D’Hont J, Haigh JJ, Roy F, Hengel J: Efficient and User-Friendly Pluripotin-based Derivation of Mouse Embryonic Stem Cells. Stem Cell Rev 2011;8:768-778.

20 Xiong W, Gao Y, Cheng X, Martin C, Wu D, Yao S, Kim M-J, Liu Y: The use of SC1 (Pluripotin) to Support mESC Self-renewal in the Absence of LIF. J Vis Exp DOI: 10.3791/1550.

21 Loh YH, Wu Q, Chew JL, Vega VB, Zhang W, Chen X, Bourque G, George J, Leong B, Liu J et al: The Oct4 and Nanog transcription network regulates pluripotency in mouse embryonic stem cells. Nat Genet 2006;38:431-440.

22 Wang J, Rao S, Chu J, Shen X, Levasseur DN, Theunissen TW, Orkin SH: A protein interaction network for pluripotency of embryonic stem cells. Nature 2006;444:364-368.

-23 Masui S, Nakatake Y, Toyooka Y, Shimosato D, Yagi R, Takahashi K, Okochi H, Okuda A, Matoba R, Sharov AA et al: Pluripotency governed by Sox2 via regulation of Oct3/4 expression in mouse embryonic stem cells. Nat Cell Biol 2007;9:625-635.

24 Chan KK, Zhang J, Chia NY, Chan YS, Sim HS, Tan KS, Oh SK, Ng HH, Choo AB: KLF4 and PBX1 directly regulate NANOG expression in human embryonic stem cells. Stem Cells 2009;27:2114-2125.

25 Pera M, Soncin F, Mohamet L, Ritson S, Hawkins K, Bobola N, Zeef L, Merry CLR, Ward CM: E-Cadherin Acts as a Regulator of Transcripts Associated with a Wide Range of Cellular Processes in Mouse Embryonic Stem Cells. PLoS One 2011;6:e21463.

26 Hawkins K, Mohamet L, Ritson S, Merry CLR, Ward CM: E-cadherin and, in its Absence, N-cadherin Promotes Nanog Expression in mES Cells via STAT3 Phosphorylation. Stem Cells 2012;30:1842-1851.

27 Chen HF CC, Lee WC, Huang HP, Wu HC, Ho HN, Chen YJ, Kuo HC.: Surface Marker Epithelial Cell Adhesion Molecule and E-cadherin Facilitate the Identification and Selection of Induced Pluripotent Stem Cells. Stem Cell Rev 2011;7:722-735.

28 Spencer HL, Eastham AM, Merry CL, Southgate TD, Perez-Campo F, Soncin F, Ritson S, Kemler R, Stern PL, Ward CM: E-cadherin inhibits cell surface localization of the pro-migratory 5T4 oncofetal antigen in mouse embryonic stem cells. Mol Biol Cell 2007;18:2838-2851. 


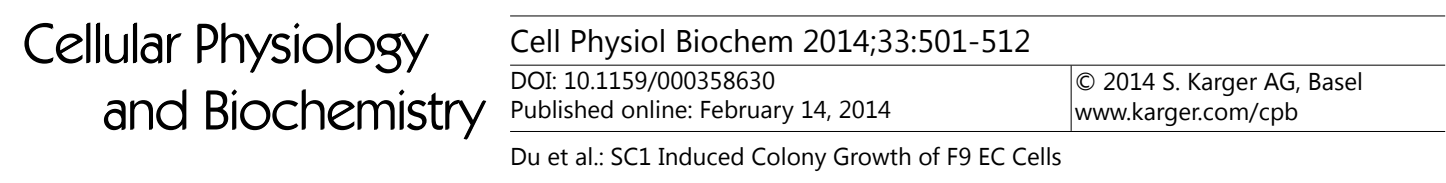

29 Eastham AM, Spencer H, Soncin F, Ritson S, Merry CLR, Stern PL, Ward CM: Epithelial-Mesenchymal Transition Events during Human Embryonic Stem Cell Differentiation. Cancer Res 2007;67:11254-11262.

- 30 Ullmann U, In't Veld P, Gilles C, Sermon K, De Rycke M, Van de Velde H, Van Steirteghem A, Liebaers I: Epithelial-mesenchymal transition process in human embryonic stem cells cultured in feeder-free conditions. Mol Hum Reprod 2006;13:21-32.

-31 Li L, Wang S, Jezierski A, Moalim-Nour L, Mohib K, Parks RJ, Retta SF, Wang L: A unique interplay between Rap1 and E-cadherin in the endocytic pathway regulates self-renewal of human embryonic stem cells. Stem Cells 2010;28:247-257.

-32 Chou YF CH, Eijpe M, Yabuuchi A, Chenoweth JG, Tesar P, Lu J, McKay RD, Geijsen N: The Growth Factor Environment Defines Distinct Pluripotent Ground States in Novel Blastocyst-Derived Stem Cells. Cell 2008;135:449-461.

33 Horie M, Ito A, Kiyohara T, Kawabe Y, Kamihira M: E-cadherin gene-engineered feeder systems for supporting undifferentiated growth of mouse embryonic stem cells. J Biosci Bioeng 2010;110:582-587.

34 Nagaoka M, Koshimizu U, Yuasa S, Hattori F, Chen H, Tanaka T, Okabe M, Fukuda K, Akaike T: E-CadherinCoated Plates Maintain Pluripotent ES Cells without Colony Formation. Plos One 2006;1:e15.

-35 Larue L, Ohsugi M, Hirchenhain J, Kemler R: E-cadherin null mutant embryos fail to form a trophectoderm epithelium. Proc Natl Acad Sci USA 1994;91:8263-8267.

-36 Redmer T, Diecke S, Grigoryan T, Quiroga-Negreira A, Birchmeier W, Besser D: E-cadherin is crucial for embryonic stem cell pluripotency and can replace OCT4 during somatic cell reprogramming. EMBO Rep 2011;12:720-726.

-37 Chen T, Yuan D, Wei B, Jiang J, Kang J, Ling K, Gu Y, Li J, Xiao L, Pei G: E-Cadherin Mediated Cell-Cell Contact Is Critical for Induced Pluripotent Stem Cell Generation. Stem Cells 2010;28:1315-1325.

-38 Wu Y, Guo Z, Wu H, Wang X, Yang L, Shi X, Du J, Tang B, Li W, Zhang Y: SUMOylation represses Nanog expression via modulating transcription factors Oct4 and Sox2. PLoS One 2012;7:e39606.

39 Takahashi K, Yamanaka S: Induction of pluripotent stem cells from mouse embryonic and adult fibroblast cultures by defined factors. Cell 2006;126:663-676.

40 Behrens J, Lowrick O, Klein-Hitpass L, Birchmeier W: The E-cadherin promoter: functional analysis of a G.C-rich region and an epithelial cell-specific palindromic regulatory element. Proc Natl Acad Sci USA 1991;88:11495-11499.

-41 Sineva GS, Pospelov VA: Inhibition of GSK3 $\beta$ enhances both adhesive and signalling activities of $\beta$-catenin in mouse embryonic stem cells. Biol Cell 2010;102:549-564.

42 Nelson WJ: Regulation of cell-cell adhesion by the cadherin-catenin complex. Biochem Soc Trans 2008;36:149-155.

43 Li E, Bestor TH, Jaenisch R: Targeted mutation of the DNA methyltransferase gene results in embryonic lethality. Cell 1992;69:915-926.

44 Berstine EG, Hooper ML, Grandchamp S, Ephrussi B: Alkaline phosphatase activity in mouse teratoma. Proc Natl Acad Sci USA 1973;70:3899-3903.

45 Todaro GJ, Green H: Quantitative studies of the growth of mouse embryo cells in culture and their development into established lines. J Cell Biol 1963;17:299-313.

-46 Mitsui K, Tokuzawa Y, Itoh H, Segawa K, Murakami M, Takahashi K, Maruyama M, Maeda M, Yamanaka S: The homeoprotein Nanog is required for maintenance of pluripotency in mouse epiblast and ES cells. Cell 2003;113:631-642.

-47 Hart AH, Hartley L, Ibrahim M, Robb L: Identification, cloning and expression analysis of the pluripotency promoting Nanog genes in mouse and human. Dev Dyn 2004;230:187-198.

48 Chen Y, Du Z, Yao Z: Roles of the Nanog protein in murine F9 embryonal carcinoma cells and their endoderm-differentiated counterparts. Cell Res 2006;16:641-650.

49 Kanatsu-Shinohara M, Takehashi M, Takashima S, Lee J, Morimoto H, Chuma S, Raducanu A, Nakatsuji N, Fässler R, Shinohara T: Homing of Mouse Spermatogonial Stem Cells to Germline Niche Depends on $\beta 1$ Integrin. Cell Stem Cell 2008;3:533-542. 